\title{
Yunpi-Huoxue-Sanjie Formula Alleviates Diabetic Cardiomyopathy by Inducing Autophagy
}

\section{Xin Zhang ( $\nabla$ drjanson@126.com )}

ZCMU: Zhejiang Chinese Medical University https://orcid.org/0000-0001-8956-6024

Jia-Qi Guan

Zhejiang Chinese Medical University

Li-Yan You

Zhejiang Chinese Medical University

Zhu-Jun Mao ( $\square$ maozhujun0107@163.com )

Zhejiang Chinese Medical University

Lu-Ping Qin ( $\sim$ qinsmmu@126.com )

Zhejiang Chinese Medical University

\section{Research}

Keywords: Yunpi-Huoxue-Sanjie formula, diabetic cardiomyopathy, autophagy

Posted Date: April 13th, 2021

DOI: https://doi.org/10.21203/rs.3.rs-213895/v2

License: (c) (i) This work is licensed under a Creative Commons Attribution 4.0 International License.

Read Full License 


\title{
Yunpi-Huoxue-Sanjie formula alleviates diabetic cardiomyopathy by inducing autophagy
}

\author{
Xin Zhang*, Jia-Qi Guan, Li-Yan You, Zhu-Jun Mao*, Lu-Ping Qin*
}

College of Pharmaceutical Sciences, Zhejiang Chinese Medical University, 548 Binwen Road, Hangzhou 310053, Zhejiang, China.

*Corresponding author: drjanson@126.com; maozhujun0107@163.com; qinsmmu@126.com

\begin{abstract}
Background: Yunpi-Huoxue-Sanjie (YP-SJ) formula is a Chinese herbal formula with unique advantages for the treatment of diabetic cardiovascular complications, such as diabetic cardiomyopathy (DCM). The aim of this study was to investigate the role of YP-SJ formula in regulating myocardial autophagy. To determine whether YPSJ formula can be used as an adjuvant therapy for DCM, its therapeutic effects and mechanism in myocardial injury caused by type 2 diabetic mellitus (T2DM) rats were determined.
\end{abstract}

Methods: A high-fat diet combined with low-dose streptozotocin (STZ) injection was used to induce DCM in rats. Biochemical reagents were used to analyze blood glucose levels, an enzyme-linked immunosorbent assay determined insulin levels, echocardiography assessed cardiac structure and function, histopathology was used to detect myocardial inflammation and fibrosis, myocardial autophagosomes were observed by transmission electron microscopy, and quantitative RT-PCR and Western blot analyses were used to detect the expression levels of autophagy-related proteins. 
Results: After treatment with YP-SJ formula, DCM rats' metabolism was abnormal and myocardial inflammation, fibrosis, and cardiac dysfunction were significantly improved. In addition, compared with DCM, YP-SJ formula significantly increased the number of autophagosomes, the expression of forkhead box protein O1 (FoxO1) and autophagy related 12 (Atg12); the expression of phosphorylated FoxO1 and p62 was significantly decreased, and the level of myocardial autophagy was increased. Conclusions: YP-SJ formula can alleviate the myocardial damage caused by T2DM, and thus may serve as an alternative treatment for DCM.

Keywords: Yunpi-Huoxue-Sanjie formula, diabetic cardiomyopathy, autophagy

\section{Introduction}

Diabetic cardiomyopathy (DCM) is a specific cardiomyopathy that occurs in diabetic patients, manifesting mainly as abnormal myocardial structure and function. It is independent of coronary heart disease, hypertension, valvular heart disease, and other heart diseases and is one of the main causes of death in diabetic patients [1]. The occurrence of type 2 diabetic mellitus (T2DM) is accompanied by myocardial damage, which causes myocardial diastolic dysfunction in asymptomatic diabetic patients, insulin resistance (IR), hyperglycemia, and hyperinsulinemia. T2DM also leads to myocardial cytotoxicity, microangiopathy, metabolic disorders, and oxidative stress, and contributes to the occurrence of DCM [2, 3]. Hypoglycemia may increase the risk of cardiovascular events [4]. Currently, there is no effective treatment for DCM.

Autophagy is the process of clearing cells of damaged organelles or debris. It can reduce the damage to cells or organelles caused by harmful metabolites and accelerate the decomposition of free fatty acids and advanced glycosylation products, thereby 
improving diabetic myocardial ischemia and metabolic disorders in diabetes.

Maintaining myocardial cell homeostasis and improving cardiac function in diabetes are of great significance [5]. IR interferes with the normal metabolism of cardiomyocytes, causing cardiomyocyte dysfunction and myocardial fibrosis, whereas autophagy can improve myocardial energy metabolism and protect the normal function of cardiomyocytes. Autophagy alleviates myocardial cell damage caused by IR and T2DM [6]. Therefore, activating autophagy and insulin-signaling pathways, improving insulin sensitivity in diabetic patients, and maintaining an appropriate level of autophagy are important ways to prevent and treat DCM.

Treatment with traditional Chinese medicine (TCM) can alleviate the progression of diabetes and its complications [7]. In fact, more than 200 traditional medicinal plants and their biologically active compounds have anti-diabetic properties, as confirmed by a large number of screening methods [8]. The TCM Yunpi-HuoxueSanjie (YP-SJ) formula is composed of five natural medicines: Atractylodes Macrocephala Koidz. (Chinese name: Baizhu), Citrus aurantium L. (Chinese name: Zhiqiao), Trichosanthes kirilowii Maxim. (Chinese name: Tianhuafen), Ostreae Concha (Chinese name: Muli), and Eupolyphaga sinensis Walker (Chinese name: Tubiechong) (Fig. 1). Atractylodes macrocephala and Fructus Aurantii accelerate the metabolism of sugar and lipids, Trichosanthes and raw oysters clear away heat and nourish yin, while the beetle accelerates blood flow and both prevents and treats cardiovascular complications in diabetes. YP-SJ formula has been used to treat DM. In our previous randomized controlled clinical trial of YP-SJ formula in combination with other oral hypoglycemic agents to treat DM, we found improvement of IR and vascular inflammation in the combined treatment group relative to the control group [9]. In animal experiments, YP-SJ formula has been shown to increase serum nitric 
oxide (NO) levels and reduce serum interleukin-6 levels by activating the phosphoinositide kinase/Akt/endothelial NO synthase pathway [10].

DCM is a serious complication of diabetes and one of the main causes of sudden death. However, the role of YP-SJ formula in ameliorating the effects of DCM are unknown. In this study, we used a rat model of DCM to evaluate the mechanism by which YP-SJ formula inhibits DCM. A brief flowchart of the study design is provided in Figure 2.

\section{Materials and Methods}

\subsection{Preparation of YP-SJ formula}

Atractylodes Macrocephala Koidz., Citrus aurantium L., Trichosanthes kirilowii Maxim. granula, Ostreae Concha granula, Eupolyphaga sinensis Walker granula, according to "Chinese Pharmacopoeia" (2015 Edition) and internal control quality standards were purchased from Jiangyin Tianjiang Pharmaceutical Co., Ltd. (Jiangyin, China). The Atractylodes Macrocephala Koidz., Citrus aurantium L., Trichosanthes kirilowii Maxim., Ostreae Concha, and Eupolyphaga sinensis Walker were prepared at a ratio of 5:2:3:10:2 in terms of crude drug content.

\subsection{High-performance liquid chromatography}

\subsubsection{Chromatographic conditions}

The chromatographic conditions were as follows.

Hesperidin: mobile phase, methanol-0.1\% phosphoric acid solution $(36: 64, \mathrm{v} / \mathrm{v})$; flow rate, $1.0 \mathrm{~mL} / \mathrm{min}$; column temperature, $25^{\circ} \mathrm{C}$; ultraviolet detection wavelength, $286 \mathrm{~nm}$; and injection volume, $20 \mu \mathrm{L}$. 
Atractylodin: mobile phase, acetonitrile-water $(70: 30, \mathrm{v} / \mathrm{v})$; flow rate, $1.0 \mathrm{~mL} / \mathrm{min}$; column temperature, $35^{\circ} \mathrm{C}$; ultraviolet detection wavelength, $340 \mathrm{~nm}$; and injection volume, $20 \mu \mathrm{L}$.

Hypoxanthine: mobile phase, methanol- $0.1 \%$ phosphoric acid solution $(2: 98, \mathrm{v} / \mathrm{v})$; flow rate, $0.5 \mathrm{~mL} / \mathrm{min}$; column temperature, $25^{\circ} \mathrm{C}$; ultraviolet detection wavelength, $254 \mathrm{~nm}$; and injection volume, $20 \mu \mathrm{L}$.

Cucurbitacins B: mobile phase, methanol-0.1\% phosphoric acid solution (53:47, $\mathrm{v} / \mathrm{v})$; flow rate, $1.0 \mathrm{~mL} / \mathrm{min}$; column temperature, $25^{\circ} \mathrm{C}$; ultraviolet detection wavelength, $254 \mathrm{~nm}$; and injection volume, $20 \mu \mathrm{L}$.

Taurine: mobile phase, methanol-0.05 M sodium acetate solution (40:60, v/v); flow rate, $0.8 \mathrm{~mL} / \mathrm{min}$; column temperature, $25^{\circ} \mathrm{C}$; ultraviolet detection wavelength, 330 $\mathrm{nm}$; and injection volume, $20 \mu \mathrm{L}$.

A mixture was made of sodium borate buffer $(0.6 \mathrm{M} ; 3.72 \mathrm{~g}$ boric acid and $2.1 \mathrm{~g}$ sodium hydroxide), derivatization reagent (0.1 g o-phthalaldehyde), $10 \mathrm{~mL}$ chromatographic methanol, and $0.1 \mathrm{~mL}$ mercaptoethanol.

\subsubsection{Sample processing and preparation}

Hesperidin, atractylodin, hypoxanthine, cucurbitacins B, and taurine ( $0.2 \mathrm{~g}$ each) were separately added to different 5 -mL centrifuge tubes with chromatographic methanol, and sonicated for $15 \mathrm{~min}$. Then $0.2 \mathrm{~mL}$ solution was filtered using a $0.22-\mu \mathrm{m}$ filter, and the filtrate was collected and centrifuged at $18000 \mathrm{r} / \mathrm{min}$ for $5 \mathrm{~min}$. Finally, $20 \mu \mathrm{L}$ of the supernatants for each compound was used for high-performance liquid chromatography (HPLC).

\subsection{Animal model and drug administration}


Thirty male Wistar rats (specific-pathogen-free) aged 8 weeks and weighing 180-200 g (Animal License No. SCXK (hu) 2017-0005; Shanghai Slake Experimental Animal Co., Ltd., Shanghai, China) were used, according to the principle of 3R used for the humane care of experimental animals. The rats were maintained under conditions of $22-25^{\circ} \mathrm{C}$, humidity of $40-70 \%$ and a $12-\mathrm{h}$ dark/12-h light cycle, and allowed to adapt to the laboratory environment for 1 week before the experiment. The rats were randomly divided into control $(n=10)$ and DCM model $(n=20)$ groups. The rats in the control group were fed a normal diet and those in the DCM model group were fed a high-fat diet (HFD, D12451; Research Diets, Inc., New Brunswick, NJ, USA). After 3 weeks of feeding, the rats in the DCM model group were intraperitoneally injected with $30 \mathrm{mg} / \mathrm{kg}$ streptozotocin (STZ, freshly prepared in citrate buffer, $0.655 \mathrm{~g}$ citric acid, $0.552 \mathrm{~g}$ sodium citrate in $100 \mathrm{~mL} \mathrm{ddH2O,} \mathrm{pH}$ 4) [11], whereas rats in the control group were intraperitoneally injected with the same volume of citric acid buffer. In the 6th week, the blood of fasting rats was collected, fasting plasma glucose (FPG) and fasting plasma insulin (FINS) were detected, and homeostatic model assessment for IR (HOMA-IR) was calculated. FPG $>11.1 \mathrm{mmol} / \mathrm{L}$ and IR indicated successful modeling [12] . The DCM model group was randomly divided into untreated (DCM model, $\mathrm{n}=$ 10) and treated (YP-SJ-treated, $\mathrm{n}=10$ ) groups. According to our previous reports and clinical equivalent doses, the rats in the treated group were administered oral YP-SJ formula $1.2 \mathrm{~g} / \mathrm{kg} / \mathrm{day}$, whereas the rats in the control and untreated groups were administered the same volume of deionized water for 10 weeks. The animal experiments were conducted according to guidelines of the National Institutes of Health Guide for the Care and Use of Laboratory animals (NIH Publications \#85-23, revised in 1985). At the end of experiment, all rats underwent metabolic and functional analyses before sacrifice, and plasma and tissue samples were collected. The left ventricular 
myocardial tissue was removed immediately; some tissue was snap-frozen in liquid nitrogen at $-80^{\circ} \mathrm{C}$ for RT-PCR and Western blot analyses, and the remaining tissue was routinely processed for histopathological examination.

\subsection{Blood analyses}

During the experiment, blood was collected from the tail vein of rats after $12 \mathrm{~h}$ fasting at night. The FPG level was measured using a glucose oxidase method (Toecho Super2, Kagawa, Japan). FINS was determined using a rat/mouse insulin enzymelinked immunoassay (ELISA) Kit (R\&D Systems, Abingdon, UK). IR was estimated using HOMA-IR:[fasting plasma insulin $\times$ fasting plasma glucose]/22.5).

\subsection{Transthoracic echocardiography}

Two-dimensional echocardiography was used to determine cardiac function and heart dimensions using a high-resolution small-animal imaging system (Vevo1100; Visual Sonics, Toronto, Canada) while the rats were anesthetized with $1.5-2 \%$ isoflurane. The system was equipped with a high-frequency ultrasound probe (MS250). The measurements of left ventricular end-systolic diameter (LVESD) and left ventricular end-diastolic diameter (LVEDD) were based on the analysis of at least three separate cardiac cycles. Fractional shortening (FS) was calculated according to the following formula: FS $(\%)=[(\mathrm{LVEDD}-\mathrm{LVESD}) / \mathrm{LVEDD}] \times 100$. Ejection fraction $(\mathrm{EF} \%)$ was calculated by: $\mathrm{EF}(\%)=([\mathrm{LVEDD}-\mathrm{LVESD}] / \mathrm{LVESD}) \times 100$. Mitral valve peak flow velocity was measured in the early diastole (E) and late diastole (A), and the E/A ratio was calculated.

\subsection{Histological examination and assessment}




\subsubsection{Observation of the morphological structure of the myocardium}

Myocardial tissue was fixed in $4 \%$ paraformaldehyde, embedded in paraffin, cut into 6- $\mu \mathrm{m}$ sections, and stained with hematoxylin and eosin $(\mathrm{H} \& \mathrm{E})$ and Masson's trichome for histological analysis. The severity of myocardial pathological changes was evaluated according to the following scores established by Rezkalla et al. [13]: 0, no inflammation or suspected inflammation; 1, small degeneration, inflammation focus; 2, multiple small inflammation foci or several large inflammation foci and small necrosis foci; 3 , multiple large inflammation and necrosis foci; and 4, diffuse inflammation infiltration, necrosis, calcification. Masson's trichome staining was used to observe the volume fraction of collagen fibers. The collagen area around the blood vessels was not included in the analysis.

\subsubsection{Observation of ultrastructure and autophagosomes of cardiomyocytes}

Rat myocardial tissue was cut into $1-\mathrm{mm}^{3}$ tissue blocks, fixed in $2.5 \%$ glutaraldehyde at $4{ }^{\circ} \mathrm{C}$ for $2 \mathrm{~h}$, and washed twice with phosphate-buffered saline (PBS) at $4{ }^{\circ} \mathrm{C}$ for 15 min each. Then the tissues were stained and fixed in $1 \%$ osmium acid at $4^{\circ} \mathrm{C}$ for $1 \mathrm{~h}$, rinsed again with PBS, and stained with $2 \%$ uranyl acetate solution for $30 \mathrm{~min}$. After gradient ethanol dehydration, propylene oxide replacement, embedding with pure embedding agent, polymerization in an oven, and staining with $4 \%$ uranium acetate, transmission electron microscopy (TECNA-10; Netherlands Philips Co., Amsterdam, Netherlands) was performed.

\subsection{Analysis of mRNA expression}

Quantitative RT-PCR (qRT-PCR) was used to detect the mRNA levels of cardiac target genes. Detailed information on the primers is shown in Table 1. Reverse 
transcription was carried out according to the instructions of a reverse transcription reaction kit (Thermo Fisher Scientific, Waltham, MA, USA). The threshold cycle (CT) values were used to obtain quantitative values. The relative average fold changes in expression were calculated by the $2^{-\triangle \Delta \mathrm{CT}}$ method. All samples were run in parallel. The normalized curve method was used to measure the expression levels of forkhead box protein $\mathrm{O} 1$ (FoxO1), p62, and autophagy-related 12 (Atg12), which were normalized to GAPDH.

\subsection{Analysis of protein expression}

Heart tissue was lysed and centrifuged at $11000 \mathrm{rpm}$ for $20 \mathrm{~min}$ at $4^{\circ} \mathrm{C}$. The protein concentration was quantitatively analyzed, and the protein lysate was subjected to sodium dodecyl sulfate polyacrylamide gel electrophoresis on a $10 \%$ polyacrylamide gel, and then electrotransferred onto a PVDF membrane (Millipore, Billerica, MA, USA). Then the membrane was blocked in $5 \%$ skim milk and probed overnight at $4{ }^{\circ} \mathrm{C}$ with the following primary antibodies: FoxO1 (1:1000; Abcam, Cambridge, MA, USA), phosphorylated FoxO1 (p-FoxO1, 1:1000), p62 (1:1000), Atg12 (1:1000; Cell Signaling Technology, Danvers, MA, USA), and $\beta$-tubulin (1:5000, Lianke Biotech, Hangzhou, China). Then the membranes were incubated with rabbit or mouse secondary antibodies labeled with horseradish peroxidase, and proteins were detected by enhanced chemiluminescence (Millipore) using a VersaDoc 4000 MP workstation (Bio-Rad, Hercules, CA, USA). ImageJ software was used for density analysis to determine the relative expression levels of target proteins (Bethesda, MD, USA).

\subsection{Statistical analysis}


All data are presented as the mean \pm standard error of the mean. Statistical analysis was conducted using one-way analysis of variance followed by the Bonferroni post hoc test for multiple comparisons. $P<0.05$ was considered statistically significant.

\section{Results}

\subsection{HPLC analysis of the YP-SJ formula}

The retention time of hesperidin was about $8.898 \mathrm{~min}$. The content of hesperidin in the granules was $0.054 \pm 0.0029 \%(n=3)$; After extraction of methanol-dissolved granules, the content of hesperidin was $0.066 \pm 0.0078 \%(n=3)$. Atractylodes are highly soluble in fat and insoluble in wáter; thus, no atractylodin was detected in the aqueous extract of the Chinese herbal compound. The retention time of hypoxanthine was about $6.985 \mathrm{~min}$. The content of hypoxanthine in the granules was $0.019 \pm$ $0.00058 \%(\mathrm{n}=3)$. The retention time of Cucurbitacin B was about $23.885 \mathrm{~min}$. The content of Cucurbitacin B in the granules was $0.0022 \pm 0.00045 \%(n=3)$. After extraction of methanol-dissolved granules, the content of hesperidin was $0.0044 \pm$ $0.00025 \%(\mathrm{n}=3)$. The retention time of taurine derivatives was about $8.368 \mathrm{~min}$. The content of taurine in the granules was $0.15 \pm 0.0058 \%(n=3)$, as shown in the chromatogram Fig. 3.

\subsection{YP-SJ formula attenuates IR in DCM rats}

Compared with the control group, the FPG of rats in the untreated group increased by 4.2 fold. After YP-SJ formula treatment, compared with the untreated group, the levels were reduced by $16.6 \%$ (Fig. 4a). Before the end of the experiment, the HOMA-IR index was higher in the untreated group than in the control group. 
Compared with the untreated group, after YP-SJ formula treatment, FINS and HOMA-IR indexes were reduced by $18.1 \%$ and $33.9 \%$, respectively (Fig. 4b, c)

\subsection{YP-SJ formula improves cardiac function in DCM rats}

Echocardiography was used to analyze cardiac function. Compared with the control group, LVESD and LVEDD increased more significantly (by $31.6 \%$ and $16.0 \%$, respectively), EF (\%) and FS (\%) decreased more significantly (by $13.1 \%$ and $17.6 \%$, respectively) in the untreated group. E/A ratio $>2$ in the untreated group. Compared with the untreated group, after YP-SJ formula treatment, LVESD and LVEDD decreased more significantly (by $16.8 \%$ and $9.7 \%$, respectively), EF (\%) and FS (\%) decreased more significantly (by $15.1 \%$ and $21.3 \%$, respectively) in the treated group; E/A ratio $<2$ in the treated group, implying the administration of YP-SJ formula significantly reduced the damage of DCM (Fig. 5a-f).

\subsection{YP-SJ formula reduces myocardial inflammation and necrosis in DCM rats}

In the control group, H\&E staining of the myocardium showed mild edema of the myocardial cells and no lesions were seen (Fig. 6a). In the untreated group, myocardial cells had mild edema, vacuoles were seen in a few cells, and occasional inflammatory cell infiltration, local myocardial fiber necrosis, and fibrous hyperplasia in the lesion area were observed (Fig. 6b). Myocardial cells in the treatment group had mild edema, vacuoles were seen in a few cells, and occasional inflammatory cell infiltration was observed (Fig. 6c). The myocardial pathology scores in each group are shown in Fig. 6d.

3.5 YP-SJ formula reduces the severity of myocardial fibrosis in DCM rats 
The control group had few distributed interstitial collagen fibers, which were evenly distributed (Fig. 7a). Collagen fibers in the myocardial tissue of the untreated group were significantly increased, and the distribution was disordered and uneven (Fig. 7b). Myocardial collagen fibers in the treated group were significantly reduced compared with the untreated group (Fig. 7c). The degree of myocardial fibrosis in each group is shown in Fig. 7d.

\subsection{YP-SJ formula enhances cardiac autophagy in the myocardial tissue of DCM} rats

The control group had higher levels of cardiac autophagy (Fig. 8a). The level of autophagy in the untreated group was significantly decreased (Fig. 8b), but was significantly increased in the treated group (Fig. 8c). Quantitative analysis of the number of cardiac autophagosomes in each group is shown in Fig. 8d.

\subsection{Analysis of mRNA expression in the rat heart}

The mRNA expression levels of FoxO1, p62, and Atg12 in each group were detected by RT-PCR, and the results are shown in Fig. 9. Compared with the control group, p62 mRNA expression in the untreated group was significantly increased, and the mRNA expression of FoxO1 and Atg12 was significantly decreased. Compared with the untreated group, p62 mRNA expression was significantly decreased and the mRNA expression of FoxO1 and Atg12 was significantly increased in the treated group. The results show that YPSJ maintained or downregulated p62 mRNA expression and upregulated FoxO1 and Atg12 mRNA expression. 


\subsection{Effects of YP-SJ formula on protein expression in the heart of DCM rats}

The expression of Foxo1 and Atg12 in the myocardium of the untreated group was significantly reduced compared with the control group $(p<0.01)$, and protein expression was significantly increased in the treated group $(p<0.05)$ (Fig. 10a, b, d). Compared with the control group, the protein expression of p-Foxo $1^{\operatorname{ser} 256}$ and p62 in the myocardium of the untreated group was upregulated $(p<0.01$ and $p<0.05$, respectively). After treatment with YP-SJ formula, expression of the abovementioned proteins was significantly decreased ( $p<0.01$ and $p<0.05$, respectively) (Fig. 10a, c, e).

\section{Discussion}

In the traditional medical practice of various ethnic groups, many plants and minerals have been used for their anti-diabetic effects, especially T2DM. The medicinal composition of YP-SJ formula is: Atractylodes Macrocephala Koidz., Citrus aurantium L., Trichosanthes kirilowii Maxim., Ostreae Concha, and Eupolyphaga sinensis Walker. The compatibility of Atractylodes Macrocephala Koidz. and Citrus aurantium L. is called Zhizhu Pills. This is from "The Theory of Spleen and Stomach", written in 1249 AD. They have the effect of promoting food digestion and the metabolism of sugar and lipids [14]. The combination of Trichosanthes kirilowii Maxim. and Ostreae Concha is called Gualoumuli powder, from "Synopsis of Prescriptions of the Golden Chamber", which was written in about 205 AD. Gualoumuli powder is currently used for the treatment of diabetes and has the effect of clearing heat and nourishing yin [15]. Eupolyphaga sinensis Walker has been used in TCM for more than 2000 years; it is popular, thought to be good, and, can activate the blood. According to the theory of TCM, the pathogenesis underlying DCM is 
spleen deficiency, yin deficiency, fire exuberance, and blood stasis. YP-SJ formula has shown efficacy in treating these deficiencies, giving it a clinical basis for the treatment of DCM.

IR occurs when the body compensates for excessive insulin secretion to maintain stable blood sugar, and may cause metabolic syndrome and T2DM. Our previous study found that YP-SJ formula particles significantly improved FPG, FINS, HOMAIR index, and other indicators in patients with T2DM, and reduced vascular inflammation [9]. Our previous study also showed that YP-SJ formula effectively improved glucose metabolism in DCM rats. Compared with the control group, IR in the untreated group was significantly worsened, but was alleviated in the treated group [10].

Diabetes can worsen the structure and function of the heart, and may lead to heart failure even without coronary atherosclerosis and high blood pressure. Early manifestations are left ventricular diastolic dysfunction, characterized by reduced left ventricular filling speed and late diastolic filling patterns. During disease progression, left ventricular systolic dysfunction occurs with reduced ejection fraction [16]. Structurally, this leads to left ventricular hypertrophy with fibrosis, possibly due to replacement of apoptotic and/or necrotic cardiomyocytes by connective tissue [17]. The development of fibrosis leads to myocardial stiffness and impaired contractility, resulting in systolic and diastolic dysfunction in late DCM due to increased left ventricular wall mass and thickness. This leads to four clinical phenotypes [18] : the first stage is diastolic dysfunction with normal ejection fraction, the second stage is combined systolic and diastolic dysfunction, the third stage is non-obstructive microvascular disease or systolic and diastolic dysfunction of coronary atherosclerosis, and the fourth stage is obvious ischemia or infarction that can lead to 
heart failure. In this study, the myocardium of the DCM model group showed obvious signs of inflammation, necrosis, and fibrosis. At the same time, the systolic function indexes FS and EF were decreased and the diastolic function index E/A was > 2, implying impaired myocardial contraction and diastolic function in DCM rats. YP-SJ formula can effectively reduce myocardial inflammation, necrosis, fibrosis, and cardiac damage in DCM rats.

In DCM, IR not only induces a lack of myocardial energy supply but also leads directly to myocardial cell dysfunction and apoptosis. The results of clinical and animal experiments show that YP-SJ formula can effectively reduce blood sugar levels and improve IR. IR interferes with the normal metabolism of cardiomyocytes, causing cardiomyocyte dysfunction and myocardial fibrosis, whereas autophagy can improve myocardial energy metabolism and protect the normal function of myocardial cells. Autophagy has the effect of antagonizing myocardial cell damage caused by T2DM IR [19]. In an animal model of T2DM induced by a HFD combined with STZ, cardiomyocyte autophagy was inhibited $[20,21]$. The results showed that compared with the normal group, the number of myocardial autophagosomes in the DCM rat model was significantly reduced. YP-SJ formula effectively increased the level of myocardial autophagy in DCM rats and alleviated myocardial damage. There was an increased number of autophagosomes in the myocardium of the control group, because the basal level of autophagy controls the elimination of long-lived proteins and damaged organelles, promotes survival, and maintains the balance of intracellular metabolism. Under high-glucose conditions, autophagy is inhibited; YP-SJ formula can increase the number of autophagosomes. However, autophagy is a highly dynamic process, and the increase or decrease of autophagosomes is not completely consistent with autophagic activity. Autophagy flux determines autophagic activity, in 
that the formation of autophagosomes and the fusion of autophagosomes and lysosomes reflects overall functional autophagy. Atg12 is an important regulatory protein for closed autophagosomes that completes the extension and formation of phagocytic vesicles. In the process of lysosomal degradation, p62 bound to substrate is degraded by proteolytic enzymes, indicating that elevated levels of p62 are generally regarded as a sign of inhibited autophagy activity [3]. Therefore, in this study, we observed regulation of the autophagy protein Atg12 and the degradation of autophagy substrate p62. The results show that YP-SJ formula reduced the expression of p62, increased the expression of Atg12, increased the autophagy level of cardiomyocytes, and inhibited the damage from DCM (Fig. 11).

During IR and hyperinsulinemia, the autophagy activity and transcription levels of genes such as Atg12 are inhibited; this is closely related to transcriptional regulation mediated by the downstream signaling molecule FoxO1 [22]. FoxO1 is the main regulator of insulin signal transduction and glucose homeostasis. During starvation, FoxO1 can induce transcription of glucose 6-phosphatase and phosphoenolpyruvate carboxykinase, promote liver gluconeogenesis, and maintain stable blood sugar. However, in conditions of IR and diabetes, due to the lack of regulation of insulin signaling, overactive FoxO1 continues to promote gluconeogenesis in an uncontrolled manner, leading to hyperglycemia and ultimately participating in the occurrence of diabetes and its complications and development $[23,24]$. In cardiomyocytes, FoxO1 activation caused by IR and hyperglycemia participates in myocardial mitochondrial biogenesis and cardiac homeostasis, and promotes myocardial remodeling at least in part by increasing the expression of $\beta$ myosin heavy chain [25]. Overactivated FoxO1 can also promote the expression of inducible NO synthase in DCM, and further induce the nitrosylation of target proteins such as glyceraldehyde-3-phosphate 
dehydrogenase and caspase-3, leading to post-hyperglycemia myocardial cell death and myocardial dysfunction [26]. In terms of autophagy regulation, FoxO positively regulates autophagy and activates a variety of autophagy-related transcription factors, such as Atg5 and Atg12 [27]. In oral squamous cell carcinoma cell lines, oncogenes can inhibit the expression of FoxO1 and its downstream target genes, and reduce the level of autophagy [28]. In vivo studies in mice have shown that under cell stress conditions such as starvation or myocardial ischemia/reperfusion, it can accelerate the dephosphorylation of FoxO1, increase the nuclear transcription activity of FoxO1 transcription factors, increase the level of cardiac autophagy, and reduce cardiomyocyte hypertrophy [29]. FoxO1 is a regulatory protein of IR and myocardial autophagy. Quercetin can enhance hypoxia-induced autophagy in pulmonary arterial smooth muscle cells through the FoxO1 pathway [30]. Metformin reduces oxidative stress through the AMP-activated protein kinase (AMPK)/sirtuin 1-FoxO1 pathway and enhances autophagy in diabetic nephropathy [31]. Consistent with the abovementioned findings, in this study, we confirmed that the mRNA and protein expression of FoxO1 in the myocardium of the DCM rat model was significantly increased. YP-SJ formula inhibited p-FoxO1 and promoted the transcription of FoxO1 in the nucleus, thereby upregulating the expression of Atg12. This is a possible mechanism by which YP-SJ formula increases the level of myocardial autophagy and slows down the development of DCM (Fig. 11). The results from this study provide a better understanding of the role of YP-SJ formula in the treatment of DCM.

\section{Conclusion}

This study shows for the first time that YP-SJ formula increases the expression of FoxO1 and Atg12; decreases the expression of phosphorylated FoxO1 and p62; 
increases myocardial cell autophagy; and alleviates myocardial damage caused by T2DM. These findings may help to better understand the role of YP-SJ formula in the treatment of DM and its complications as well as its potential mechanism of action.

\section{Abbreviations}

Atg12: autophagy related 12; DCM: diabetic cardiomyopathy; EF: ejection fraction; ELISA: enzyme-linked immunoassay; FINS: fasting plasma insulin; FoxO1: forkhead box protein O1; FPG: fasting plasma glucose; FS: fractional shortening; HFD: highfat diet; HOMA-IR: homeostatic model assessment for IR; IR: insulin resistance; LVESD: left ventricular end-systolic diameter; LVEDD: left ventricular end-diastolic diameter; NO: nitric oxide; qRT-PCR: quantitative RT-PCR; STZ: streptozotocin; T2DM: type 2 diabetic mellitus.

\section{Acknowledgements}

Not applicable.

\section{Author' contributions}

Xin Zhang generated the data, wrote the manuscript and approved its final version. Xin Zhang, Zhu-Jun Mao and Lu-Ping Qin designed the study. Xin Zhang, Jia-Qi Guan and Li-Yan You carried out the experiments. All authors have read and approved the final manuscript.

\section{Funding}

This study was supported by the grants from the National Natural Science Foundation of China (No. 81904110). 


\title{
Availability of data and materials
}

The datasets used in this study are available from the corresponding author upon reasonable request.

\section{Ethics approval and consent to participate}

Not applicable.

\section{Consent for publication}

Not applicable.

\section{Competing interests}

The authors declare that they have no competing interests.

\author{
Author details \\ College of Pharmaceutical Sciences, Zhejiang Chinese Medical University, 548 Binwen Road, \\ Hangzhou 310053, Zhejiang, China.
}

\section{References}

1. Gulsin Gaurav S, Athithan Lavanya, McCann Gerry P. Diabetic cardiomyopathy: prevalence, determinants and potential treatments. Ther Adv Endocrinol Metab. 2019; 10: 2042018819834869. https ://doi.org/10.1177/2042018819834869.

2. Bersch-Ferreira Ângela Cristine, Sampaio Geni Rodrigues, Gehringer Marcella Omena, et al. Association between plasma fatty acids and inflammatory markers in patients with and without insulin resistance and in secondary prevention of cardiovascular disease, a cross-sectional study. Nutr J. 2018; 17: 26. https ://doi.org/10.1186/s12937-018-0342-1.

3. Zhang Yi, Mun Su Ran, Linares Juan F, et al. ZZ-dependent regulation of p62/SQSTM1 in autophagy. Nat Commun. 2018; 9(1): 4373. https ://doi.org/0.1038/s41467-018-06878-8. 
4. Maack Christoph, Lehrke Michael, Backs Johannes, et al. Heart failure and diabetes: metabolic alterations and therapeutic interventions: a state-of-the-art review from the Translational Research Committee of the Heart Failure Association-European Society of Cardiology. Eur Heart J. 2018; 39(48): 4243-4254. https ://doi.org/10.1093/eurheartj/ehy596

5. Chaanine Antoine H. Autophagy and Myocardial Remodeling: Is it Autophagy or Autophagic Machinery and Signaling Pathways Regulating it? J Am Coll Cardiol. 2018; 71(18): 2011-2014. https ://doi.org/10.1016/j.jacc.2018.02.067.

6. Ceylan Asli F, Wang Shuyi, Kandadi Machender R, et al. Cardiomyocyte-specific knockout of endothelin receptor a attenuates obesity cardiomyopathy. Biochim Biophys Acta Mol Basis Dis. 2018; 1864(10): 33393352. https ://doi.org/10.1016/j.bbadis.2018.07.020.

7. Watal Geeta, Dhar Preeti, Srivastava Sharad Kr, et al. Herbal medicine as an alternative medicine for treating diabetes: the global burden. .Evid Based Complement Alternat Med. 2014; 2014: 596071. https ://doi.org/10.1155/2014/596071.

8. Yang L, Wu L, Wang D, et al. Role of endoplasmic reticulum stress in the loss of retinal ganglion cells in diabetic retinopathy. Neural Regeneration Research. 2013; 8(33): 3148-3158. https ://doi.org/10.3969/j.issn.1673-5374.2013.33.009.

9. Zhang Xin, MAO Zhujun. Effect of Yunpi-Huoxue-Sanjie formula on insulin resistance and vascular inflammation in patients with type 2 diabetes. Heilongjiang Traditional Chinese Medicine. 2018; 47: 61-62. https ://doi.org/CNKI:SUN:HLZY.0.2018-03-031.

10. MAO Zhu-jun, Zhang Xin, Zhang Lu, et al. Yunpi Huoxue Sanjie decoction inhibited vascular endothelial injury of type 2 diabetic rats by regulating PI3K/Akt/eNOS pathway. China Journal of Traditional Chinese Medicine and Pharmacy. 2020; 35(9): 4672-4675.

11. Mansor L S, Gonzalez E R, Cole M A, et al. Cardiac metabolism in a new rat model of type 2 diabetes using high-fat diet with low dose streptozotocin. Cardiovascular Diabetology. 2013; 12(1):136-136. https ://doi.org/10.1186/1475-2840-12-136.

12. Le Page Lydia M, Rider Oliver J, Lewis Andrew J, et al. Increasing Pyruvate Dehydrogenase Flux as a Treatment for Diabetic Cardiomyopathy: A Combined 13C Hyperpolarized Magnetic Resonance and Echocardiography Study. Diabetes. 2015; 64(8): 2735-2743. https ://doi.org/ doi:10.2337/db14-1560.

13. Rezkalla S, Kloner R A, Khatib G, et al. Effect of metoprolol in acute coxsackievirus B3 murine myocarditis. J Am Coll Cardiol. 1988; 12(2): 412-414. https ://doi.org/10.1016/0735-1097(88)90414-7.

14. Liu Yi, Zhao Xiao-rui, Wang Rui, et al. Effect of Zhizhuwan on gastrointestinal peptide concentrations in plasma of diabetic gastroenteropathy with constipation patients. China Journal of Chinese Materia Medica. 2008; 33(24): 2966-2968. https://pubmed.ncbi.nlm.nih.gov/19294863/

15. Zhao Wen-wen, Song Yue-ren, Liu Rui-rui, et al. Analysis on the Treatment of Early Diabetes with Gualou- 
muli Powder. Journal of Traditional Chinese Medicine. 2017; 58(18): 1607-1608. https ://doi.org/10.13288/j.11-2166/r.2017.18.021.

16. Murtaza Ghulam, Virk Hafeez Ul Hassan, Khalid Muhammad, et al. Diabetic cardiomyopathy-A comprehensive updated review. Prog Cardiovasc Dis. 2019; 62(4): 315-326. https ://doi.org/10.1016/j.pcad.2019.03.003.

17. Zamora, M, Villena, J.A. Contribution of Impaired Insulin Signaling to the Pathogenesis of Diabetic Cardiomyopathy. Int J Mol Sci. 2019; 20(11). https://doi.org/10.3390/ijms20112833.

18. Mizamtsidi M, Paschou S A, Grapsa J, et al. Diabetic cardiomyopathy: a clinical entity or a cluster of molecular heart changes? European journal of clinical investigation. 2016; 46(11): 947-953. https://doi.org/10.1111/eci.12673.

19. Ceylan Asli F, Wang Shuyi, Kandadi Machender R, et al. Cardiomyocyte-specific knockout of endothelin receptor a attenuates obesity cardiomyopathy. Biochim Biophys Acta Mol Basis Dis. 2018; 1864(10): 33393352. https://doi.org/10.1016/j.bbadis.2018.07.020.

20. Kobayashi Satoru, Liang Qiangrong, Autophagy and mitophagy in diabetic cardiomyopathy. Biochim Biophys Acta. 2015; 1852(2): 252-261. https://doi.org/10.1016/j.bbadis.2014.05.020.

21. Wu Aiping, Hu Pengfei, Lin Jian, et al. Activating Cannabinoid Receptor 2 Protects Against Diabetic Cardiomyopathy Through Autophagy Induction. Front Pharmacol. 2018; 9: 1292. https://doi.org/10.3389/fphar.2018.01292.

22. Duan Peng, Wang Jinxin, Li Yang, et al. Opening of mitoKATP improves cardiac function and inhibits apoptosis via the AKT-Foxo1 signaling pathway in diabetic cardiomyopathy. Int J Mol Med. 2018; 42(5): 2709-2719. https://doi.org/10.3892/ijmm.2018.3832.

23. Cheng Zhiyong, White Morris F, Targeting Forkhead. box O1 from the concept to metabolic diseases: lessons from mouse models. Antioxid Redox Signal. 2011; 14(4): 649-661. https://doi.org/10.1089/ars.2010.3370.

24. O-Sullivan InSug, Zhang Wenwei, Wasserman David H, et al. FoxO1 integrates direct and indirect effects of insulin on hepatic glucose production and glucose utilization. Nat Commun. 2015; 6: 7079. https://doi.org/10.1038/ncomms8079.

25. Qi Yajuan, Zhu Qinglei, Zhang Kebin, et al. Activation of Foxol by insulin resistance promotes cardiac dysfunction and $\beta$-myosin heavy chain gene expression. Circ Heart Fail. 2015; 8(1): 198-208. https://doi.org/10.1161/CIRCHEARTFAILURE.114.001457.

26. Xin Zhenlong, Ma Zhiqiang, Jiang Shuai, et al. FOXOs in the impaired heart: New therapeutic targets for cardiac diseases.[J] .Biochim Biophys Acta Mol Basis Dis. 2017; 1863(2): 486-498. https://doi.org/10.1016/j.bbadis.2016.11.023

27. Cao Qing-Hua, Liu Fang, Yang Zu-Li, et al. Prognostic value of autophagy related proteins ULK1, Beclin 1, 
ATG3, ATG5, ATG7, ATG9, ATG10, ATG12, LC3B and p62/SQSTM1 in gastric cancer. Am J Transl Res. 2016; 8(9): 3831-3847. https://pubmed.ncbi.nlm.nih.gov/27725863/

28. Zhou Lu, Guo Jihua, Jia Rong, Oncogene SRSF3 suppresses autophagy via inhibiting BECN1 expression. Biochem Biophys Res Commun. 2019; 509(4): 966-972. https://doi.org/10.1016/j.bbrc.2019.01.048.

29. Sengupta Arunima, Molkentin Jeffery D, Yutzey Katherine E. FoxO transcription factors promote autophagy in cardiomyocytes. J Biol Chem. 2009; 284(41): 28319-28331. https://doi.org/10.1074/jbc.M109.024406.

30. He Yuan-zhou, Cao Xiao-pei, Guo Pu-jian, et al. Quercetin induces autophagy via FOXO1-dependent pathways and autophagy suppression enhances quercetin-induced apoptosis in PASMCs in hypoxia. Free Radic Biol Med. 2017; 103: 165-176. https://doi.org/10.1016/j.freeradbiomed.2016.12.016

31. Ren Huiwen, Shao Ying, Wu Can et al. Metformin alleviates oxidative stress and enhances autophagy in diabetic kidney disease via AMPK/SIRT1-FoxO1 pathway. Mol Cell Endocrinol. 2020; 500: 110628. https://doi.org/10.1016/j.mce.2019.110628 


\section{Figures}

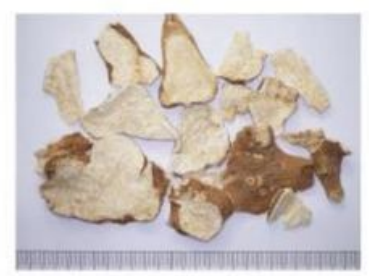

Baizhu

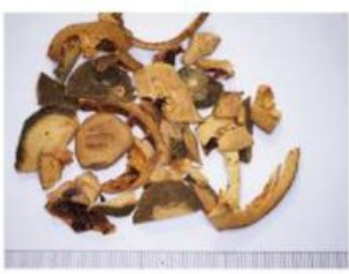

Zhiqiao

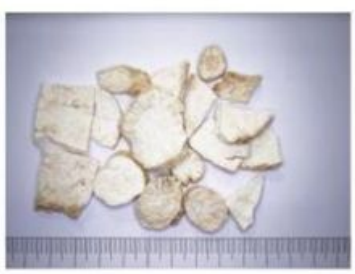

Tianhuafen

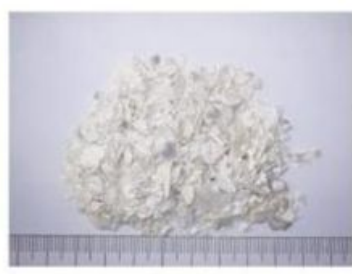

Muli

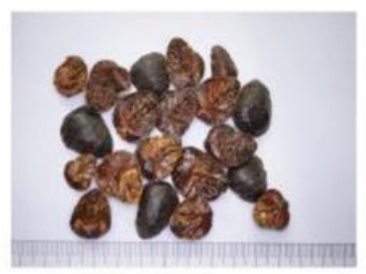

Tubiechong

Figure 1

Photographs of Atractylodes Macrocephala Koidz. (Baizhu), Citrus aurantium L. (Zhiqiao), Trichosanthes kirilowii Maxim. (Tianhuafen), Ostreae Concha (Muli) and Eupolyphaga sinensis Walker (Tubiechong) in medicinal ingredient forms 


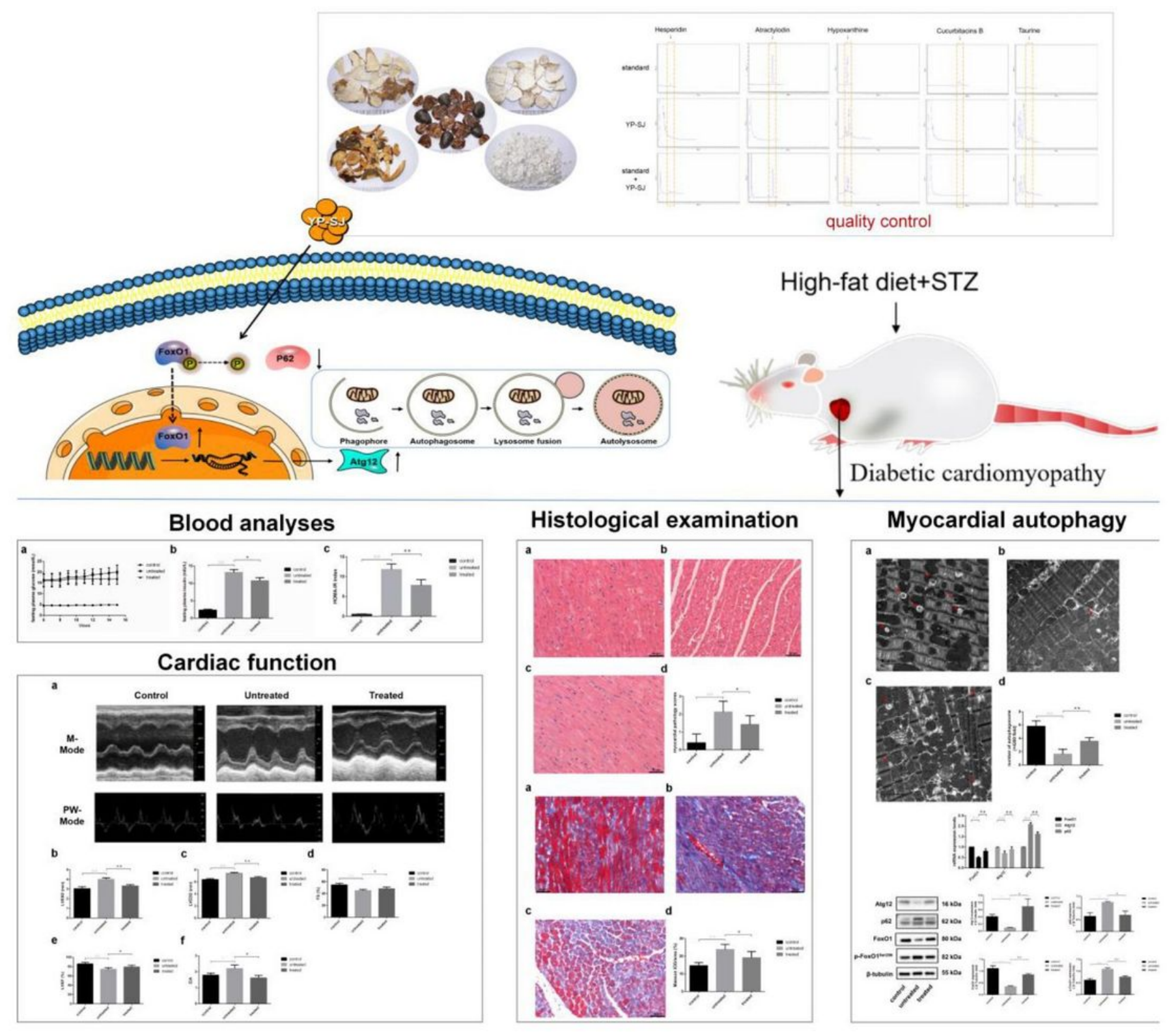

Figure 2

Flowchart of the study design that Yunpi-Huoxue-Sanjie formula alleviates diabetic cardiomyopathy by inducing autophagy 


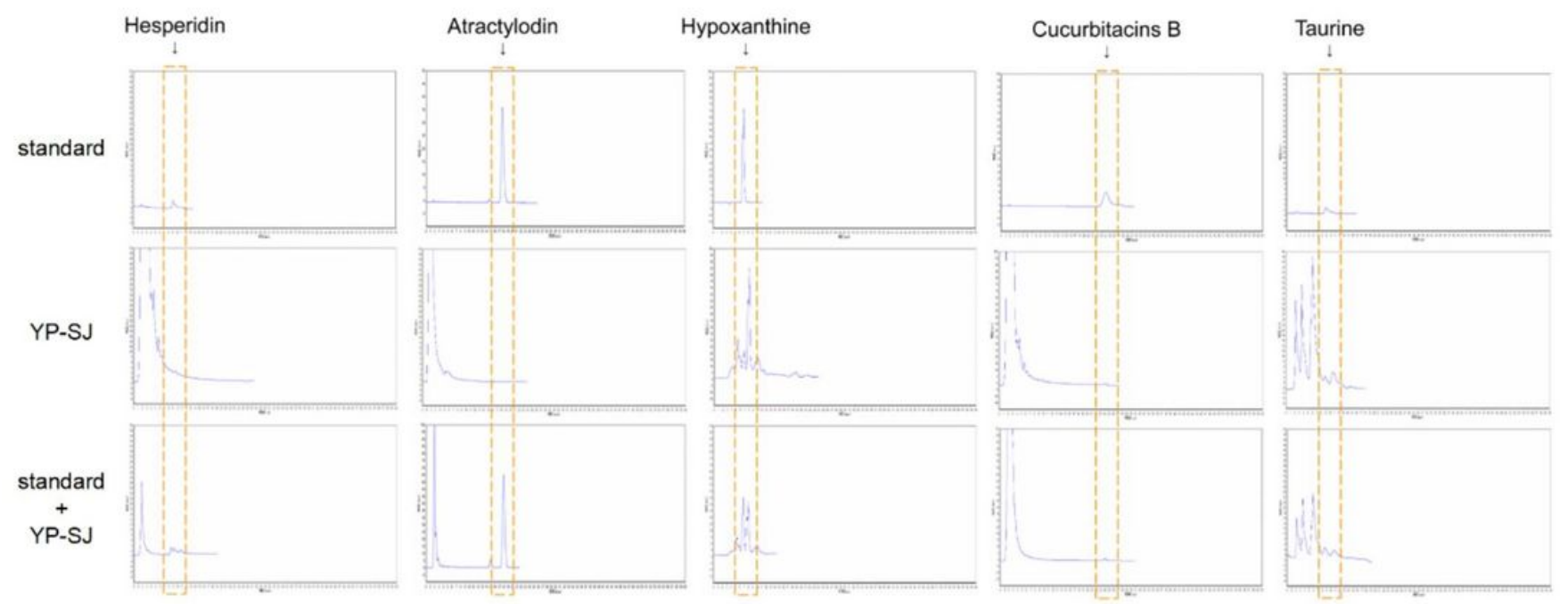

\section{Figure 3}

HPLC estimation of YP-SJ formula. The presence of hesperidin, atractylodin, hypoxanthine, cucurbitacins $\mathrm{B}$ and taurine were adopted as quality controls for YP-SJ formula. The abovementioned ingredients were detected in standard, YP-SJ and standard +YP-SJ samples

a

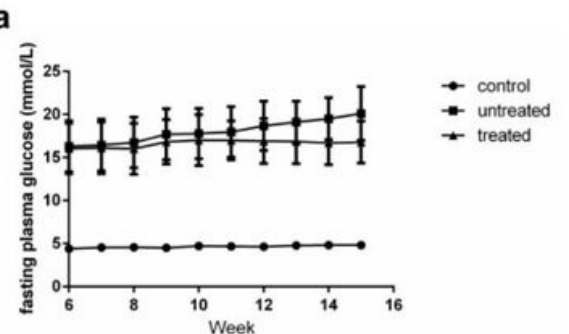

b

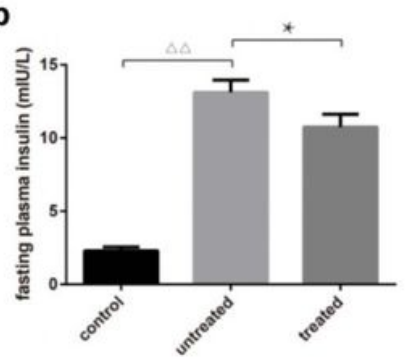

C

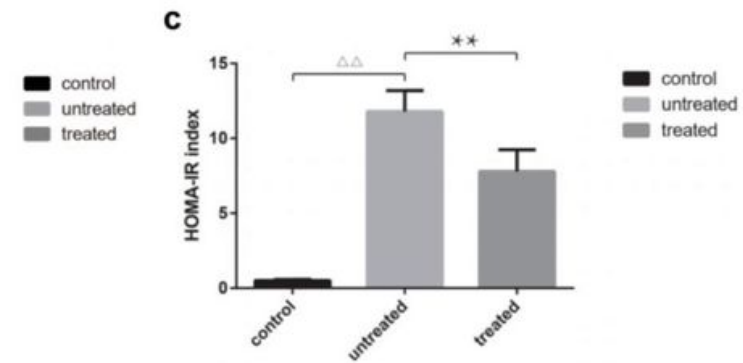

\section{Figure 4}

The effect of YP-SJ formula on a fasting plasma glucose, $b$ fasting plasma insulin and c HOMA-IR index. Data are shown by mean \pm SEM $(n=8) . \Delta \Delta p<0.01$ showing a significant difference as compared with control group; ${ }^{\star \star} p<0.01$ and ${ }^{\star} p<0.05$ showing a critical difference as compared with untreated group 

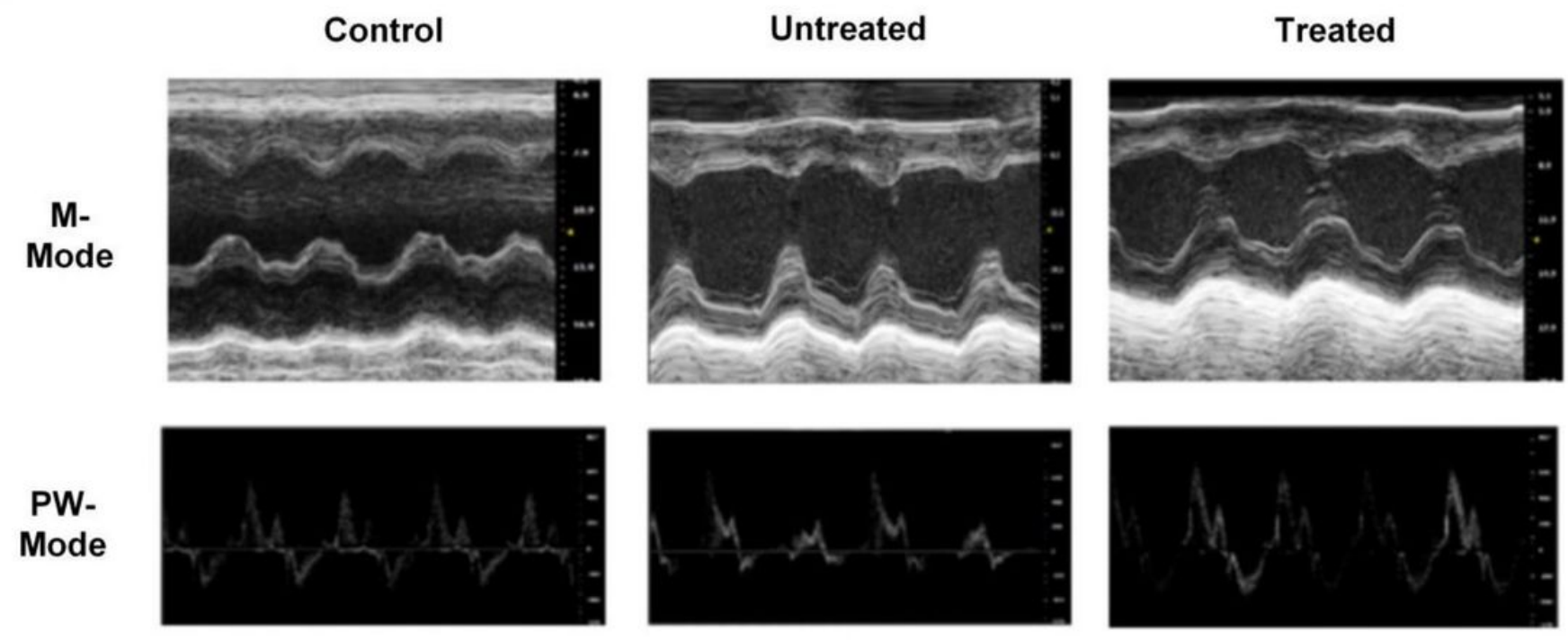

b

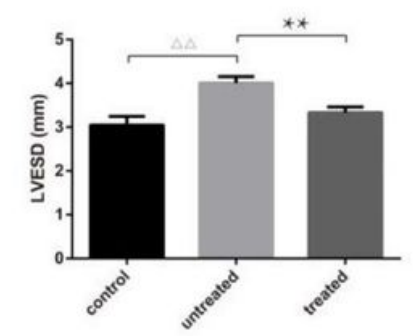

e

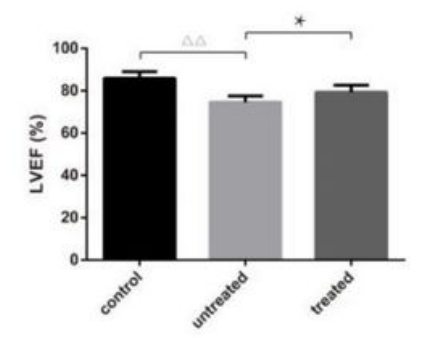

c

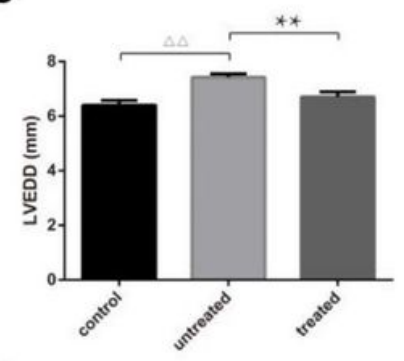

f

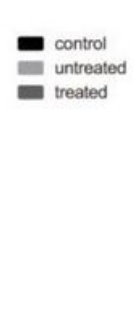

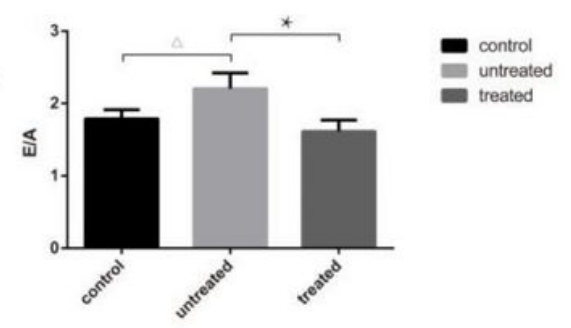

d

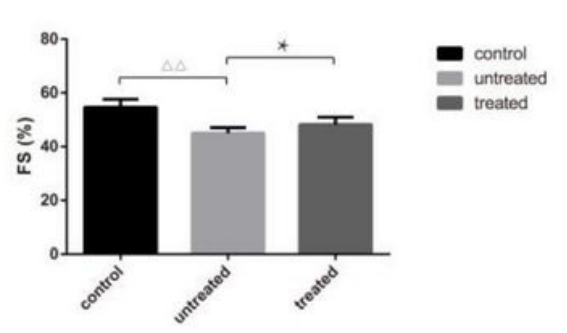

Figure 5

The effect of YP-SJ formula on cardiac function. a Representative echocardiographic images from at least three separate cardiac cycles of the hearts from control, treated and untreated rats are provided. $b$ left ventricular end-systolic diameter (LVESD) and c left ventricular end-diastolic diameter (LVEDD) were analyzed by echocardiography. $d$ The Left ventricular ejection fraction (EF), e fractional shortening (FS) and $f$ E/A ratio were calculated. $\Delta \Delta p<0.01$ and $\Delta p<0.05$ vs. control group; ${ }^{* \star} p<0.01$ and ${ }^{*} p<0.05$ vs untreated group 
a

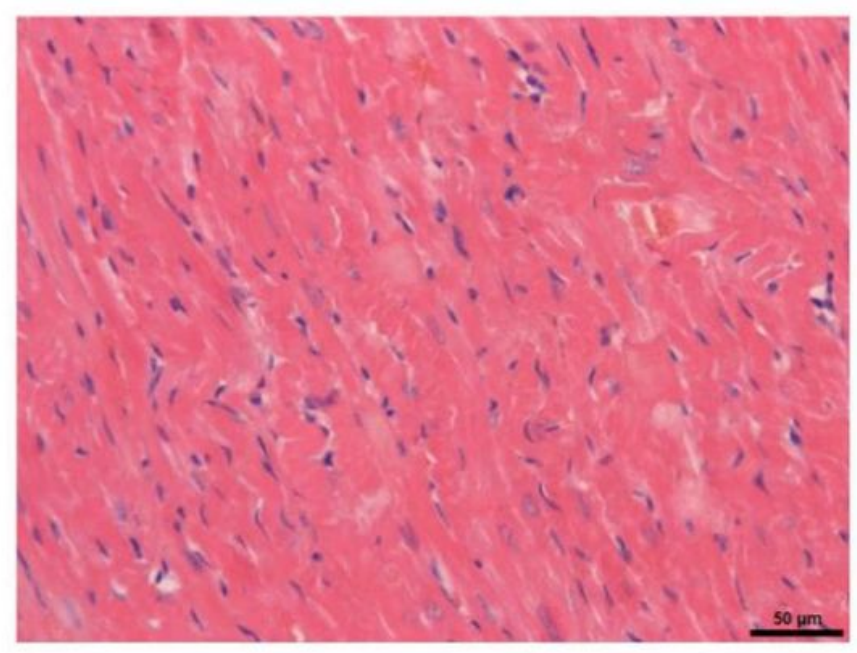

C

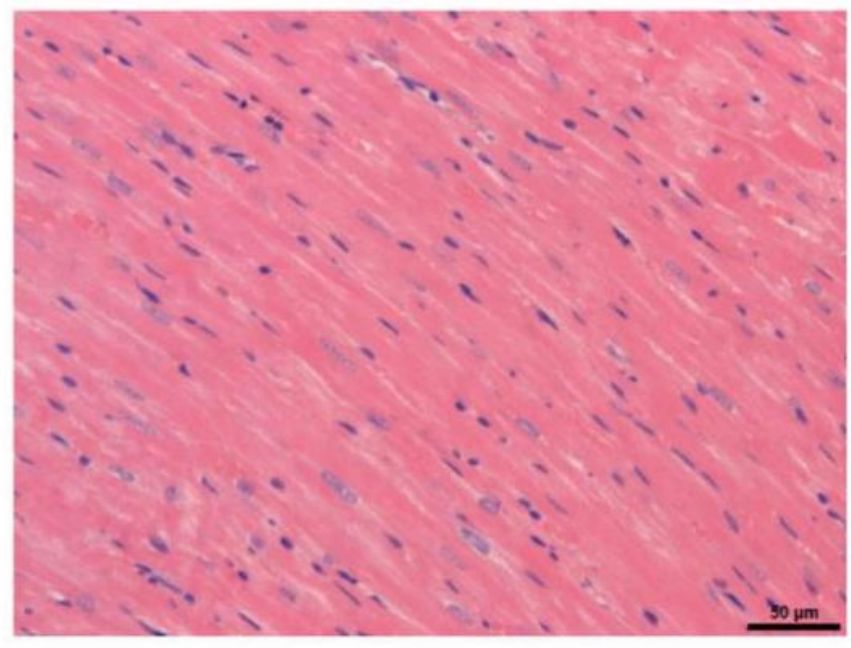

b

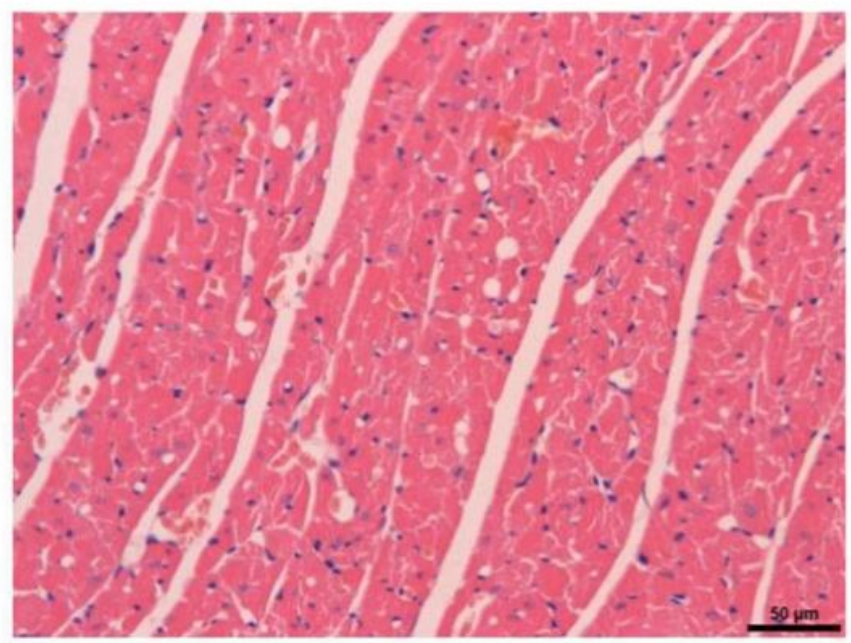

d

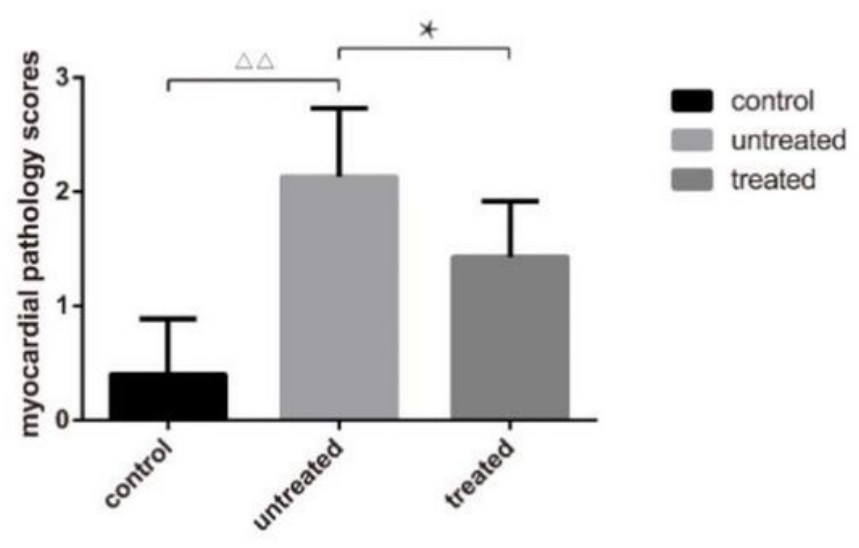

\section{Figure 6}

The effect of YP-SJ formula on the rat myocardium. Myocardial specimens $(n=5)$ were collected and

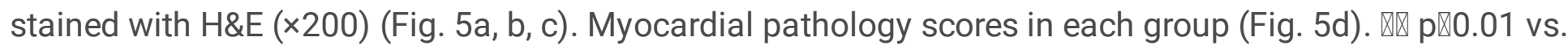

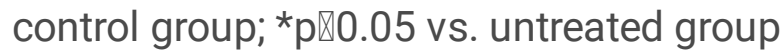


a

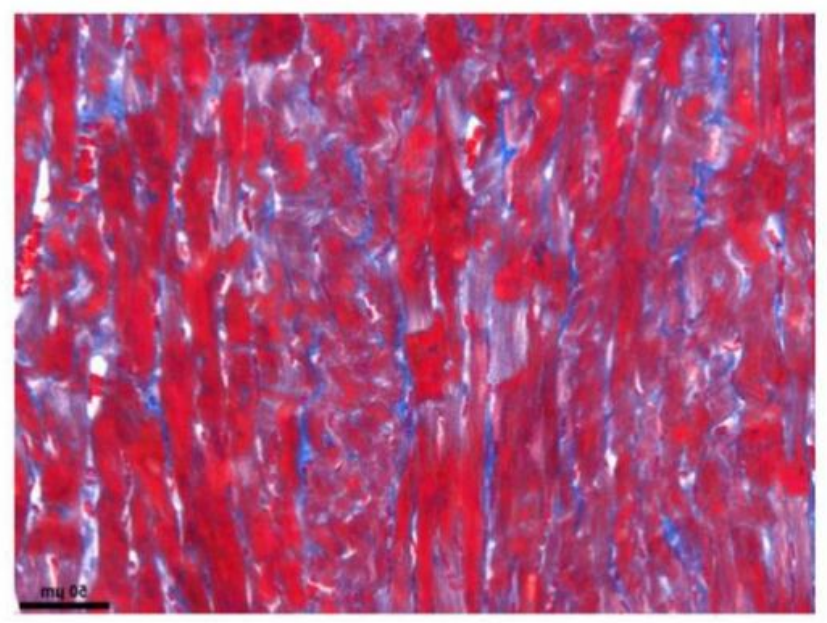

C

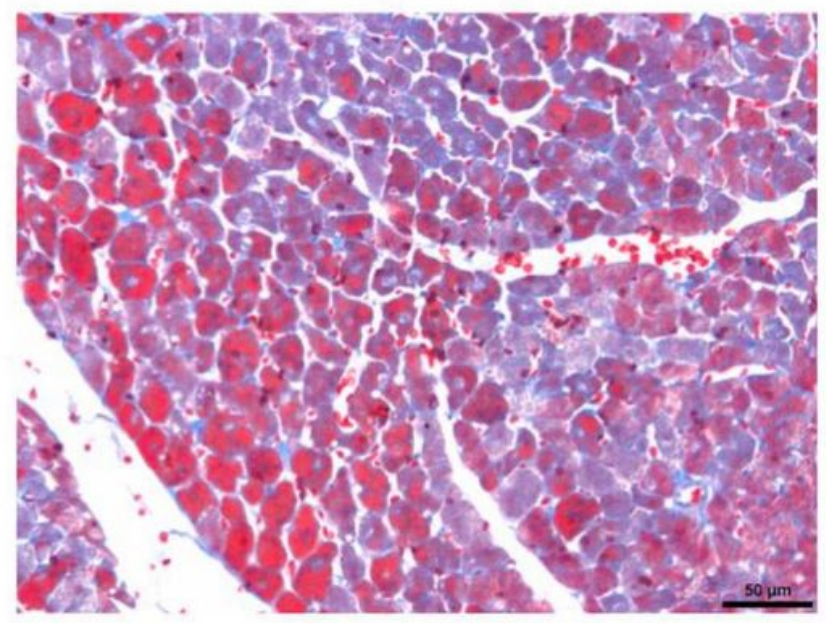

b

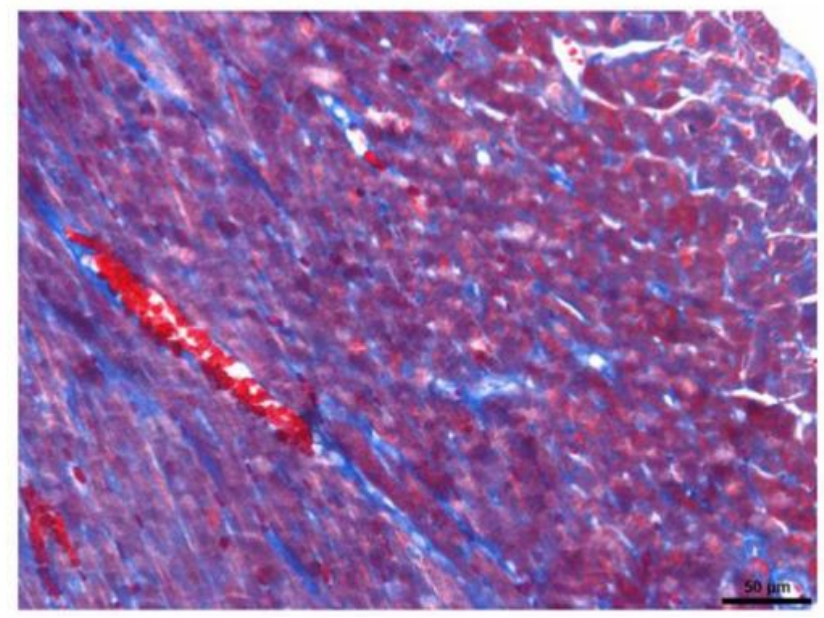

d

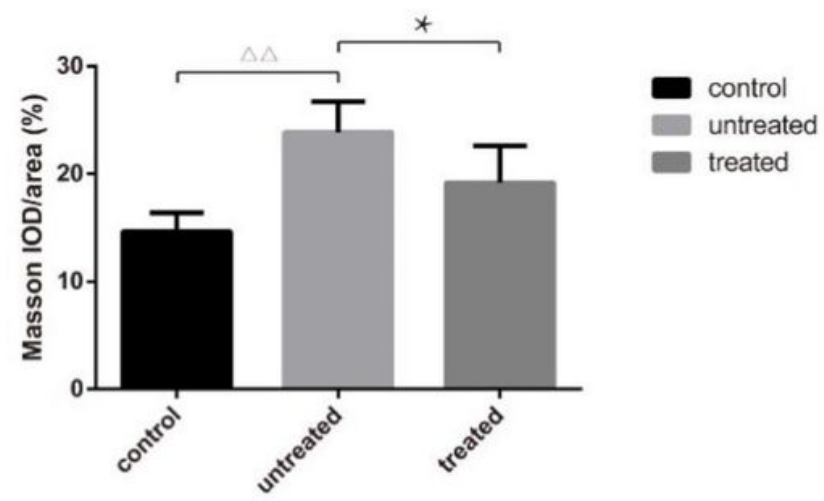

\section{Figure 7}

The effect of YP-SJ formula on the rat myocardial fibrosis. Myocardial specimens $(n=5)$ were collected and stained with Masson ( $\times 200)$ (Fig. 7a, b, c). Degree of myocardial fibrosis in each group (Fig. 7d). 怄 $p$ $₫ 0.01$ vs. control group; ${ }^{*} \mathbb{p} \otimes 0.05$ vs. untreated group 
a

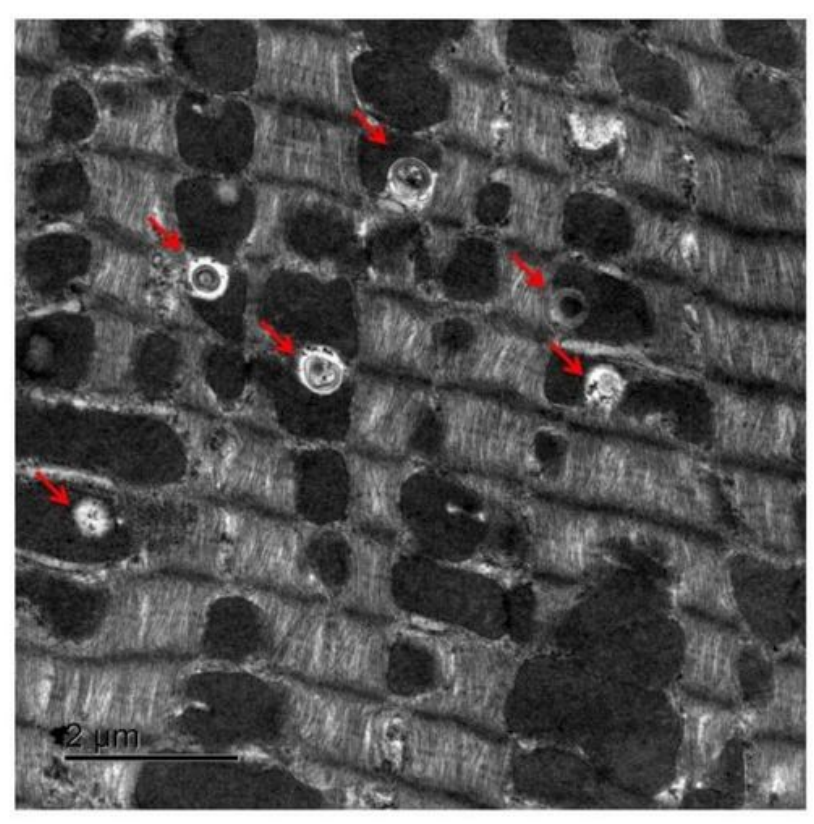

C

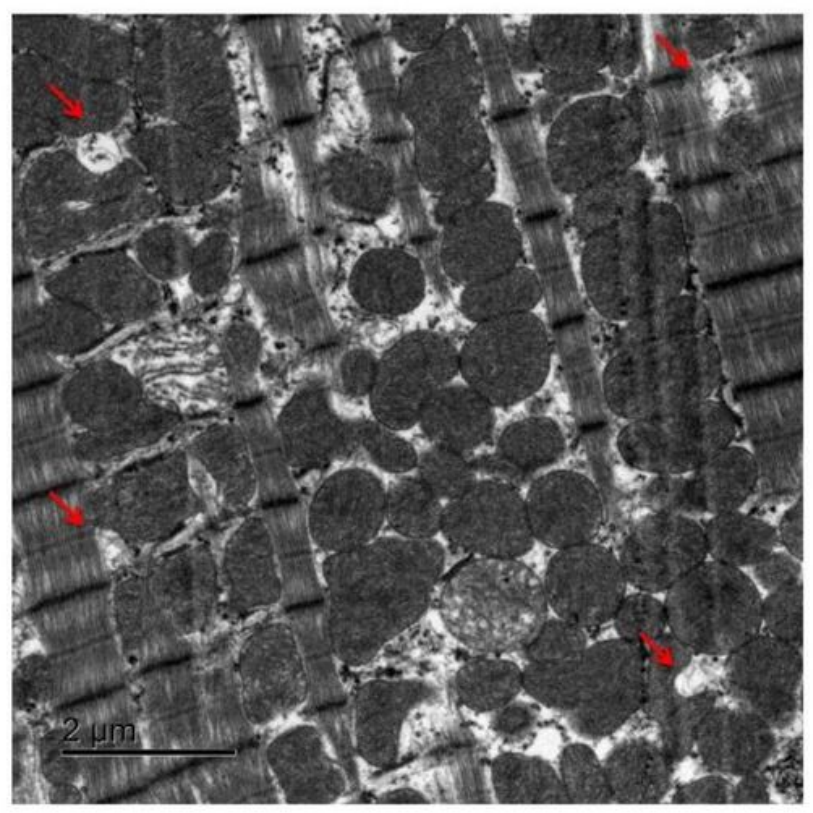

b

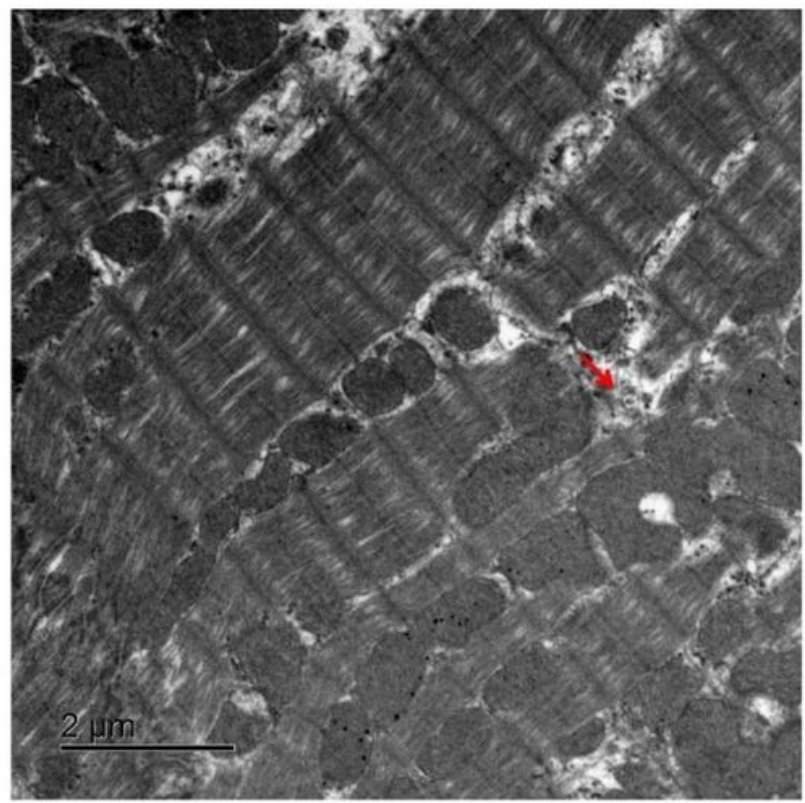

d

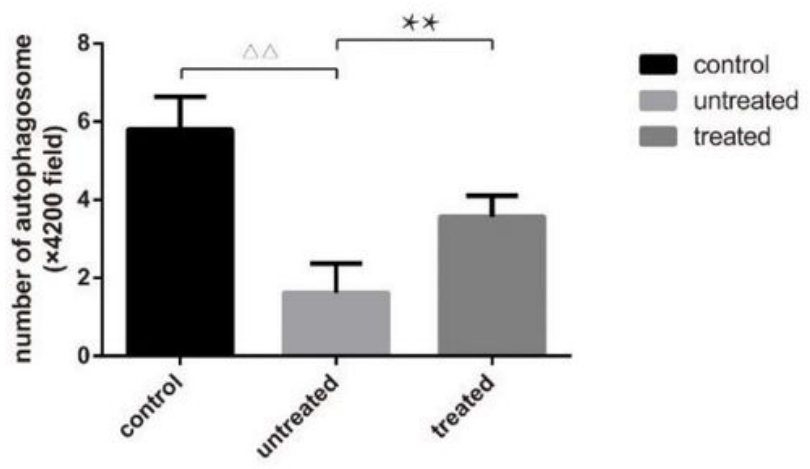

Figure 8

The effect of YP-SJ formula on the rat cardiac autophagy. Representative images of transmission electron microscopy with arrows marking autophagosomes. (×4200) (Fig. 8a, b, c). Quantitative analysis

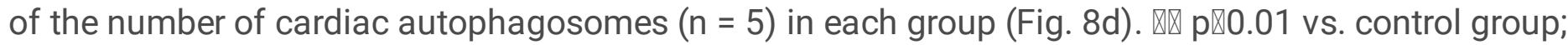
**p $₫ 0.01$ vs. untreated group 


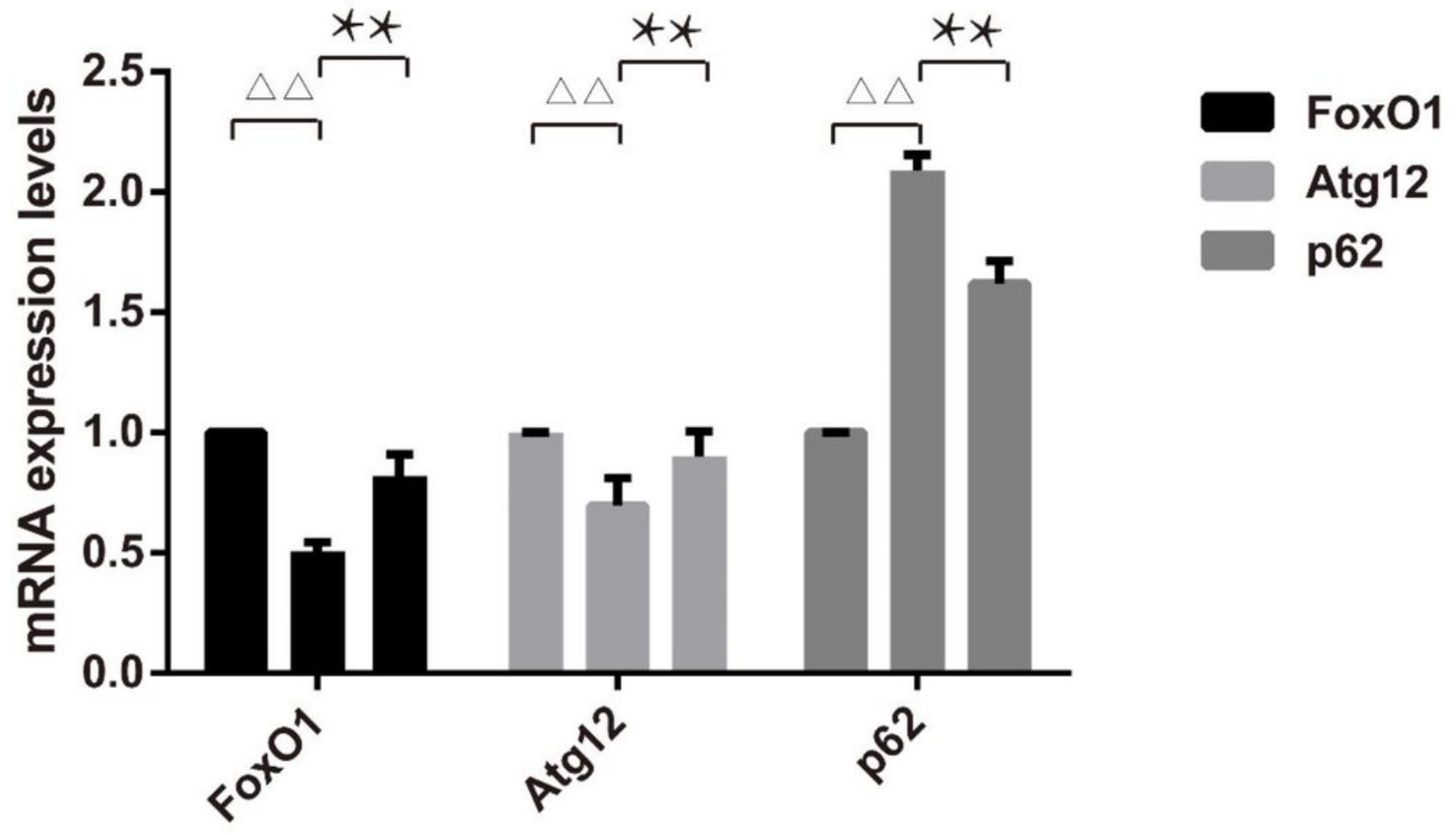

Figure 9

The effect of YP-SJ formula on gene expression. The gene expressions of Fox01, Atg12 and p62 were

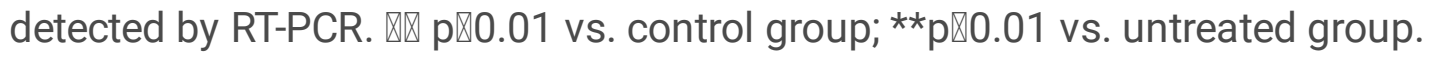


a

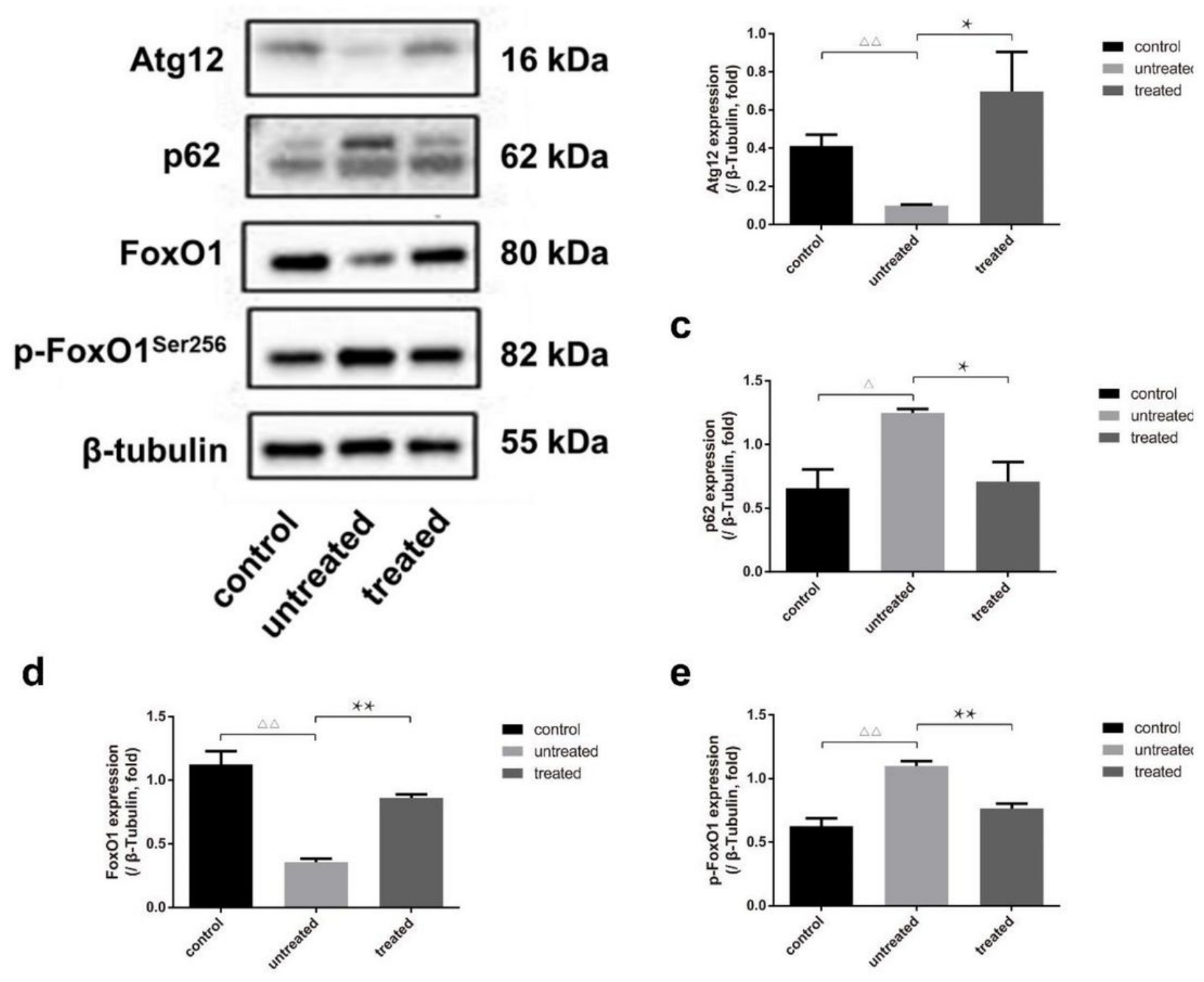

Figure 10

The effect of YP-SJ formula on protein expression. The protein expressions of Atg12, p62, Fox01 and pFox01ser256 were detected by western blot. The figure represents one of three experiments with similar results (Fig. 10a). In Fig. 10b, $\Delta \Delta p<0.01$ vs. control group, $\llbracket p<0.05$ vs. untreated group. In Fig. 10c, $\Delta p<0.05$ vs. control group, $\llbracket p<0.05$ vs. untreated group. In Fig. $10 d, \Delta \Delta p<0.01$ vs. control group, $\mathbb{\square} \otimes<<$ 0.01 vs. untreated group. In Fig. $10 \mathrm{e}, \Delta \Delta p<0.01$ vs. control group, $\mathbb{Q} \mathbb{\mathrm { Q }}<0.01$ vs. untreated group 


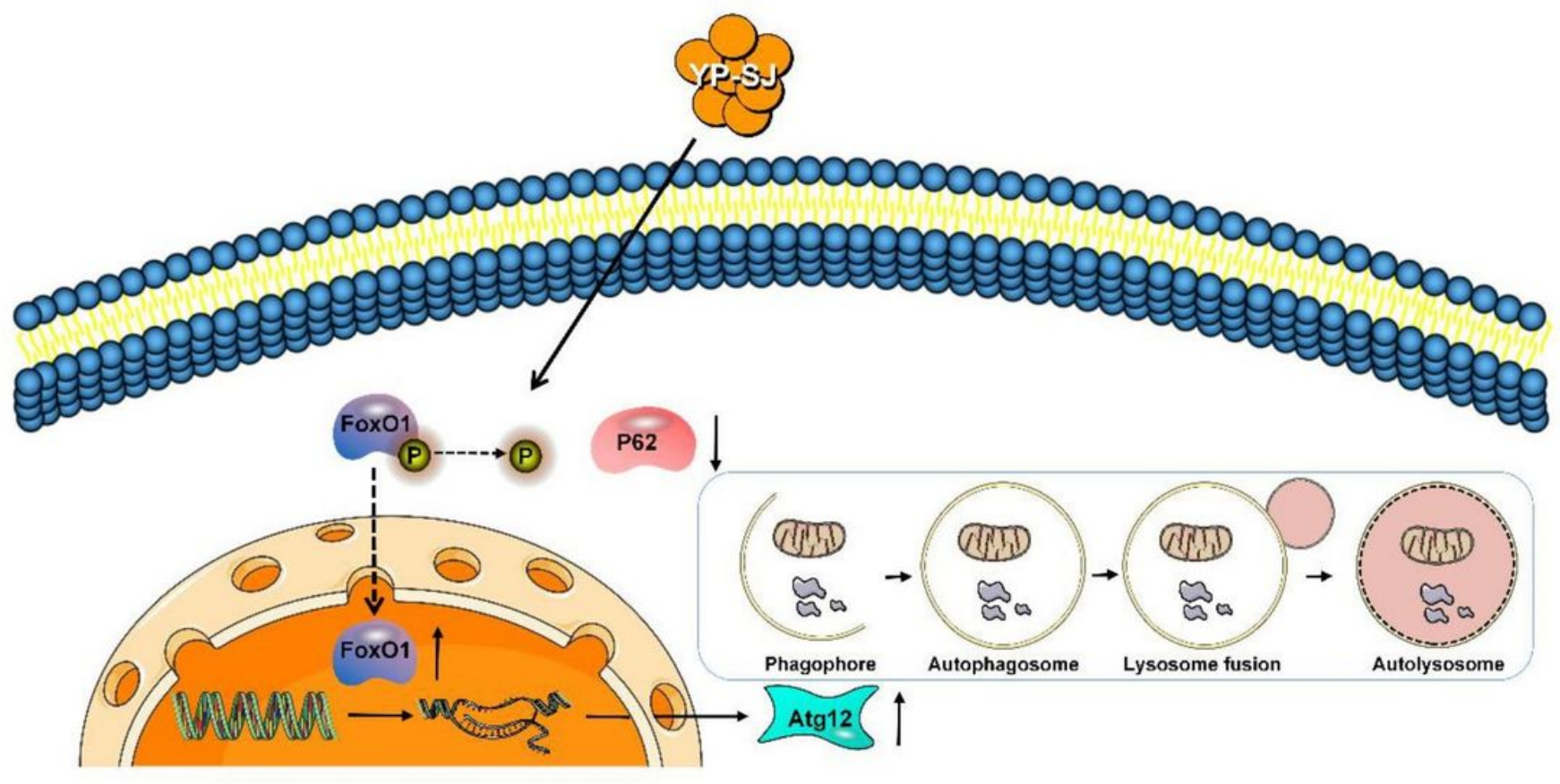

Figure 11

A schematic diagram illustrates the pathways that YP-SJ formula promotes autophagy to alleviate myocardial injury caused by type 2 diabetic mellitus 